OPEN ACCESS

Edited by:

Yixiao Luo,

Hunan Normal University, China

Reviewed by:

Tomohisa Mori,

Hoshi University, Japan

Chunxia Yan,

Xi'an Jiaotong University, China

*Correspondence:

Bin Cong

cong6406@126.com

Yingmin Li

liyingmin888@126.com

${ }^{\dagger}$ These authors have contributed equally to this work

Specialty section: This article was submitted to

Neuropharmacology,

a section of the journal

Frontiers in Neuroscience

Received: 16 October 2020 Accepted: 25 January 2021

Published: 19 February 2021

Citation:

Liu J, Yi S, Shi W, Zhang G, Wang S, Qi Q, Cong B and Li Y (2021) The Pathology of Morphine-Inhibited Nerve Repair and Morphine-Induced Nerve Damage Is Mediated via Endoplasmic Reticulum Stress. Front. Neurosci. 15:618190. doi: 10.3389/fnins.2021.618190

\section{The Pathology of Morphine-Inhibited Nerve Repair and Morphine-Induced Nerve Damage Is Mediated via Endoplasmic Reticulum Stress}

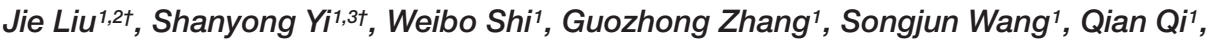 \\ Bin Cong ${ }^{1 *}$ and Yingmin $\mathrm{Li}^{1 *}$
}

1 Hebei Key Laboratory of Forensic Medicine, Collaborative Innovation Center of Forensic Medical Molecular Identification, College of Forensic Medicine, Hebei Medical University, Shijiazhuang, China, ${ }^{2}$ Research Center of Basic Medical Sciences, Department of Pathology, School of Basic Medical Sciences, Hubei University of Science and Technology, Xianning, China, ${ }^{3}$ School of Forensic Medicine, Xinxiang Medical University, Xinxiang, China

Objective: The aim of the present study was to observe the pathological damage in the cerebral cortex of rats under acute morphine exposure (AME) and different durations of morphine dependence (MD), explore whether endoplasmic reticulum stress (ERS) is involved in the damage process, and assess the effect of morphine exposure on the proliferation and differentiation of newborn neurons.

Methods: Rat models of AME and different durations of MD were established. Pathological changes in cortical neurons were assessed by hematoxylin and eosin (H\&E) and thionine staining. The expression of nuclear receptor-related factor 1 (NURR1) and that of the ERS-related proteins glucose-regulated protein 78 (GRP78), p-elF2 $\alpha$, activating transcription factor 6 (ATF6), and CHOP in cortical neurons was assessed by immunohistochemistry. Double immunofluorescence labeling was used to observe the expression of $\mathrm{Ki}-67$.

Results: H\&E and thionine staining revealed that AME resulted in pyknotic changes in cortical neurons. With prolonged morphine exposure, the number of pyknotic neurons was significantly increased, the protein expression of Ki-67 and NURR1 was significantly decreased, and the protein levels of GRP78, p-elF2 $\alpha$, ATF6, and CHOP showed marked dynamic changes.

Conclusion: AME and different durations of MD caused varying degrees of pathological changes in the cortex. Furthermore, the dynamic changes observed in ERS-related protein expression suggested that ERS may be associated with cortical injury. Different durations of MD inhibited the proliferation, differentiation, and migration of newborn neurons, which may affect the nerve repair process after injury.

Keywords: morphine dependence, acute morphine exposure, cortex, ERS, Ki67, NURR1 


\section{INTRODUCTION}

A report by the World Health Organization on the abuse of morphine indicated that the misuse of this drug had increased in recent years (Volkow and Skolnick, 2012). Morphine dependence (MD) refers to a chronic recurrent brain disease characterized by the loss of self-control and compulsive, continuous drug seeking (Lamb and Ginsburg, 2018). Several studies have shown that MD can inflict varying degrees of injury on different systems of the body, including damage to the heart and lungs, direct effects on the central nervous system, and inhibitory effects on the respiratory center. Moreover, these effects may lead to acute or chronic cerebral ischemia and hypoxia, which, in turn, may cause nerve damage (Brailoiu et al., 2004; Luo et al., 2013; Rohbani et al., 2019). MD involves a complex series of pathophysiological changes in multiple brain regions, including, importantly, the cerebral cortex (Katebi et al., 2013; Adedayo et al., 2018); however, little is known about the pathomorphological changes induced by the misuse of this substance.

The endoplasmic reticulum (ER) is the primary organelle for protein synthesis, glycosylation, folding, secretion, and nascent protein transport. Under normal conditions, the protein folding ability of the ER matches the body's protein synthesis requirements (Iurlaro and Muñoz-Pinedo, 2016; Kropski and Blackwell, 2018). However, when the body is stimulated by ischemia, hypoxia, injury, or other insults, the microenvironment of the ER changes, which can result in the accumulation of unfolded or misfolded proteins in the ER lumen and the subsequent induction of endoplasmic reticulum stress (ERS) (Xiang et al., 2017; Muneer and Shamsher Khan, 2019). Under ERS, the expression of glucose-regulated protein 78 (GRP78) increases, which can inhibit the synthesis of cellular proteins, accelerate the degradation of misfolded or unfolded proteins, and maintain ER homeostasis (Ibrahim et al., 2019). However, when ERS persists, protein kinase RNA (PKR)-like endoplasmic reticulum kinase (PERK), and activating transcription factor 6 (ATF6) can dissociate from GRP78, thereby activating downstream signaling pathways. Recent studies have shown that the activation of the ATF6 signaling pathway mainly exerts a cell-protective role (Hillary and FitzGerald, 2018), whereas the activation of the PERK/p-eIF2 $\alpha$ signaling pathway can lead to the upregulation of CCAAT/-enhancer-binding protein homologous protein (CHOP) expression, and continuous $\mathrm{CHOP}$ expression can induce cell injury, or even cell death (Hu et al., 2019). Brain injury can induce the proliferation of endogenous neural stem cells (NSCs) and enable the newborn neurons to differentiate and migrate to the site of injury (Dai et al., 2019). Ki-67, a nuclear antigen, is often used as a marker for evaluating cell proliferation (Miller et al., 2018). Meanwhile, nuclear receptor-related factor 1 (NURR1), a member of the orphan nuclear receptor superfamily, is indispensable for neuronal differentiation, migration, maturation, and survival (Dong et al., 2016).

The activation status of ERS-related proteins and the proliferation and differentiation status of endogenous NSCs in acute morphine exposure (AME)- and chronic MD-induced cortical nerve damage remain unknown. In the present study, we first established rat models of AME as well as of different durations of MD. Subsequently, we investigated the pathological changes in cortical neurons, the alterations in the expression levels of ERS-related proteins, and changes in the proliferation, differentiation, and maturation status of newborn neurons aiming to provide pathomorphological evidence for the mechanisms underlying morphine-induced injury.

\section{MATERIALS AND METHODS}

\section{Animals}

Adult male Wistar rats (Experimental Animal Center, Hebei Medical University, China), weighing $250 \pm 20$ g, were maintained in a room with an ambient temperature of $22 \pm 2^{\circ} \mathrm{C}$ and a 12/12-h light/dark cycle, and had ad libitum access to food and water. This study was approved by the Institutional Review Board for Animal Experiments at the Hebei Medical University. Every attempt was made to reduce the number of animals used and to minimize animal pain and suffering. The rats were randomly divided into the following groups: 1 -week control (Con), 3-week control, 6-week control, 1-week MD, 3-week MD, 6-week MD, 2-h control, and AME groups ( $n=8$ per group).

\section{Model of MD}

As previously described (Shi et al., 2019), the model of MD was established through subcutaneous injections of morphine hydrochloride at increasing doses. Rats in the three morphinedependence groups were subcutaneously injected in the back with morphine hydrochloride twice daily (08:00 and 20:00) for 5 days. The initial dose administered was $10 \mathrm{mg} / \mathrm{kg}$ and was increased by $10 \mathrm{mg} / \mathrm{kg}$ every other day until day 5 of treatment. The control rats received equal volumes of saline. The MD of model rats was confirmed after 5 days of morphine administration as described in Maldonado et al. (1992). Two rats were randomly selected from each control group and each morphine-dependent group and given a subcutaneous injection of naloxone hydrochloride $(5 \mathrm{mg} / \mathrm{kg})$ to induce withdrawal symptoms. Scoring involved observing signs of opiate withdrawal, including wet-dog shakes, stretching, cleaning fur, swallowing, standing, jumping, and teeth chattering. Following this assessment, $30 \mathrm{mg} / \mathrm{kg}$ morphine was administered twice daily (08:00 and 20:00) until 1-, 3-, or 6-weeks postestablishment of dependence.

Rats in the AME group were subcutaneously injected once in the back with morphine hydrochloride at a dose of $30 \mathrm{mg} / \mathrm{kg}$. The control rats received an equal volume of saline.

\section{Tissue Preparation}

Two hours after the last morphine or saline injection, the rats were deeply anesthetized and euthanized. The tissue used for staining was harvested and immediately fixed in $10 \%$ formalin, subsequently dehydrated using a graded ethanol series, and embedded in paraffin. Brain slices were obtained using a stereotaxic atlas (Paxinos and Watson, 2007) and a rotary microtome (Leica RM2255, Shanghai, China). Sections $(5 \mathrm{~mm})$ were prepared for thionine, immunohistochemical, 
and immunofluorescence staining and examined under a light microscope (Olympus IX71; Olympus, Tokyo, Japan).

\section{Reagents}

Rabbit polyclonal antibodies against GRP78 (ab188878), CHOP (ab179823), and NURR1 (ab176184); the mouse monoclonal antibody against MAP2 (ab11268); and the rabbit monoclonal antibody targeting Ki-67 (ab16667) were purchased from Abcam (United States). Rabbit polyclonal antibodies against p-eIF2 $\alpha$ (AF3087) and ATF6 (DF6009) were purchased from Affinity (China). The Alexa Fluor 488 donkey anti-mouse IgG $(\mathrm{H}+\mathrm{L})(1975519)$ and the Alexa Fluor 594 donkey anti-rabbit $\operatorname{IgG}(\mathrm{H}+\mathrm{L})$ (1827674) secondary antibodies were purchased from Invitrogen (United States). The immunohistochemistry kit (SP9001) was purchased from the Zhongshan Goldenbridge Biotech, China, and the morphine hydrochloride for injection was produced in the First Pharmaceutical Factory of Shenyang, China.

\section{Body Weight Measurements}

The body weight of the rats in all the groups was measured daily before treatment throughout the experiment.

\section{Hematoxylin and Eosin Staining}

Deparaffinized sections were stained with hematoxylin for $2 \mathrm{~min}$, transferred to $1 \%$ hydrochloric acid alcohol differentiation solution, and then stained with eosin for $3 \mathrm{~s}$.

\section{Thionine Staining}

Thionine staining was performed as previously described (Yi et al., 2019). Deparaffinized sections were stained with $4 \%$ thionine for $90 \mathrm{~s}$ at $60^{\circ} \mathrm{C}$, dehydrated through a graded alcohol series, and mounted with neutral gum.

\section{Immunohistochemistry}

Immunohistochemistry was performed as previously described (Yi et al., 2019). Antigen retrieval of deparaffinized sections was performed using a microwave, followed by incubation in 3\% $\mathrm{H}_{2} \mathrm{O}_{2}$ in cold methanol for $30 \mathrm{~min}$ and blocking for $30 \mathrm{~min}$ using goat serum. The tissues were then incubated overnight at $4^{\circ} \mathrm{C}$ with antibodies against GRP78 (1:200), p-eIF2 $\alpha$ (1:100), CHOP (1:200), ATF6 (1:100), and NURR1 (1:100). The next day, the tissues were incubated for $1 \mathrm{~h}$ with biotinylated secondary antibody and subsequently with horseradish peroxidase (HRP)conjugated biotin for $30 \mathrm{~min}$. Finally, DAB or AP-red was used as the chromagen. The tissues were counterstained with hematoxylin to demarcate locations in the sections. The primary antibodies were replaced with $0.01 \mathrm{mmol} / \mathrm{L} \mathrm{PBS}$ in the negative controls (data not shown).

\section{Immunofluorescence Double Staining}

Immunofluorescence was performed as previously described (Shi et al., 2019). The anti-Ki-67 antibody (1:100) was used as the first primary antibody and the anti-MAP2 antibody (1:150) as the second primary antibody. DyLight 594-conjugated
AffiniPure goat anti-rabbit Ig (1:150) and DyLight 488conjugated AffiniPure goat anti-mouse Ig (1:100) were used as the secondary antibodies.

\section{Cell Counting}

Six rats from each group were used for morphological observation. One out of every three serial sections were selected for cell counting. Following a comparison of the sections after immunohistochemical staining and double immunofluorescence staining, the numbers of positive cells were counted in a field of view at $100 \times$ magnification. Two independent observers who were blinded to the experimental conditions performed the counts and calculated the average number of positive cells.

\section{Statistical Methods}

The Kolmogorov-Smirnov test showed that the data were normally distributed in all groups $(P>0.1)$. The results are presented as mean \pm SD. The data were analyzed by oneway ANOVA. Significance was defined as $P<0.05$ for all statistical tests.

\section{RESULTS}

\section{Weight Change}

A change in body weight can be considered as a physiological indicator of morphine exposure. The weight of rats in the control group showed a marked increase after 1 week $(297.78 \pm 15.43)$, 3 weeks $(327.08 \pm 23.57)$, and 6 weeks $(369.52 \pm 28.81)$. In comparison, the weight of morphine-dependent rats was slightly decreased at 1 week $(260.08 \pm 8.18, P<0.01)$, with slower increases being recorded at 3 weeks $(280.18 \pm 13.14, P<0.05)$ and 6 weeks $(305.64 \pm 22.68, P<0.05)$ after treatment (Figure 1$)$.

\section{Hematoxylin and Eosin Staining Showed Pathological Changes in the Cerebral Cortex}

The cerebral cortices of rats in the control groups showed no pathological changes (data not shown), presenting a clear

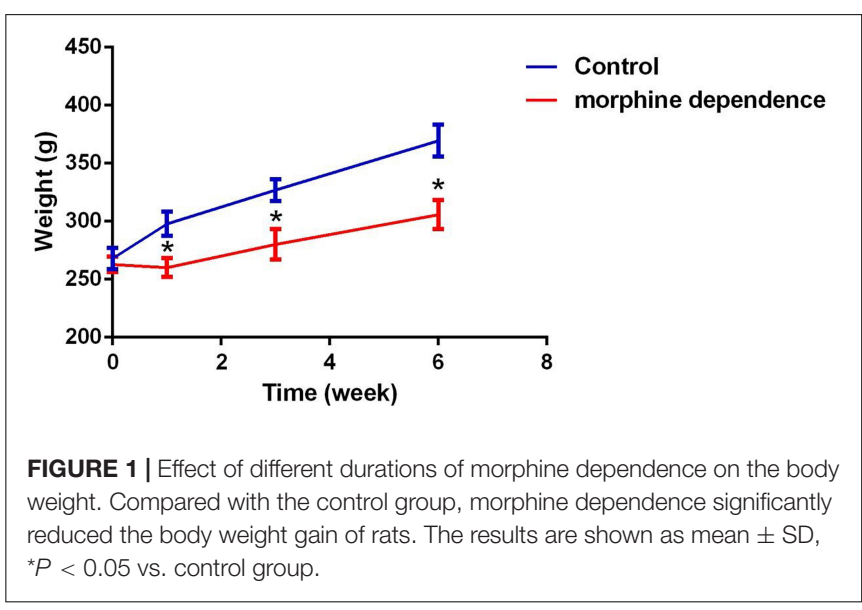


structure and neatly arranged neurons. Compared with the control group, no obvious change was observed after 1 week of MD. However, after 3 weeks of MD, the gaps around the small blood vessels and neurons widened, and microglial hyperplasia could be seen. Additionally, after 6 weeks of MD, tissue and cellular damage was more extensive and neurons were pyknotic and dying. Some pyknotic cells also appeared in the cortices of rats of the AME group (Figure 2).

\section{Thionine Staining Showed Pathological Changes in Cortical Neurons}

In the control group, the neuronal structures were clear, and Nissl bodies were evenly distributed in the cytoplasm; a similar phenotype was observed after 1 week of MD. However, after 3 weeks of MD, a proportion of Nissl bodies had disappeared and pyknotic neurons were visible. Cellular damage was more extensive at 6 weeks. Meanwhile, some Nissl bodies had also disappeared in the AME group, and pyknotic neurons were also visible (Figure 3).

\section{GRP78, p-elF2 $\alpha$, CHOP, ATF6, and NURR1 Expression in the Cerebral Cortex}

Immunohistochemical staining showed that GRP78, p-eIF2 $\alpha$, ATF6, and CHOP proteins were located in the cytoplasm and

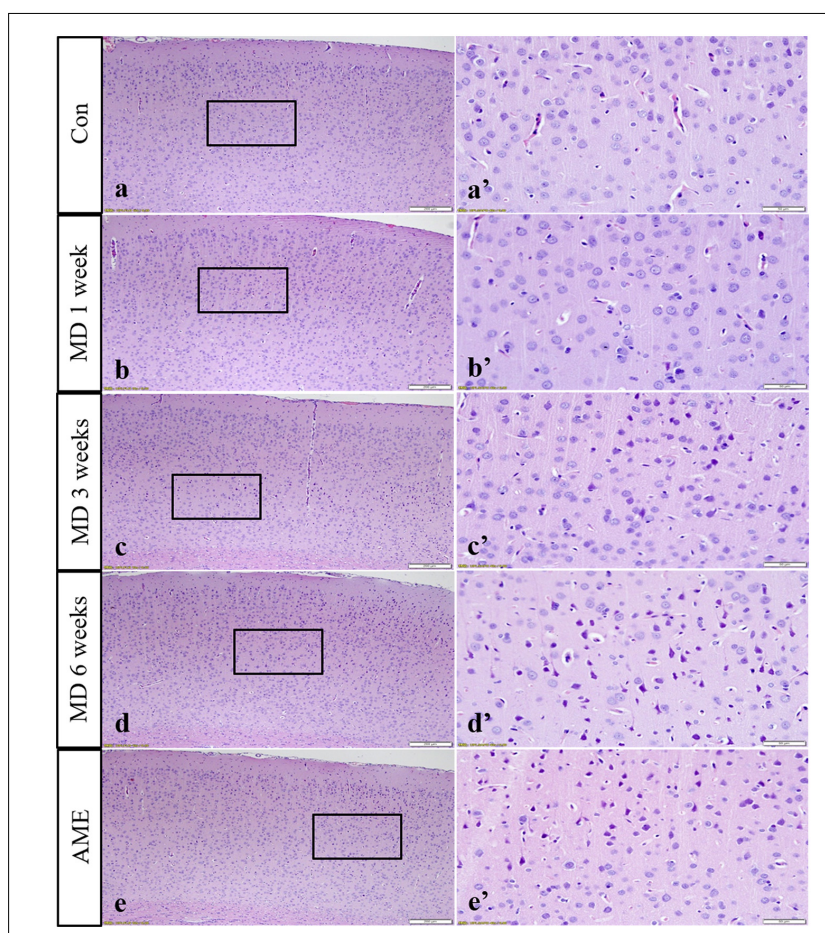

FIGURE 2 | Hematoxylin staining of the cortex. Panels (a'-e') are magnified areas of panels (a-e), respectively. With increasing periods of morphine dependence, gaps around the small blood vessels and neurons widened, and microglia hyperplasia. Bars $=200 \mu \mathrm{m}$ in panels (a-e). Bars $=50 \mu \mathrm{m}$ in panels (a'-e'); MD, morphine dependence; AME, acute morphine exposure.

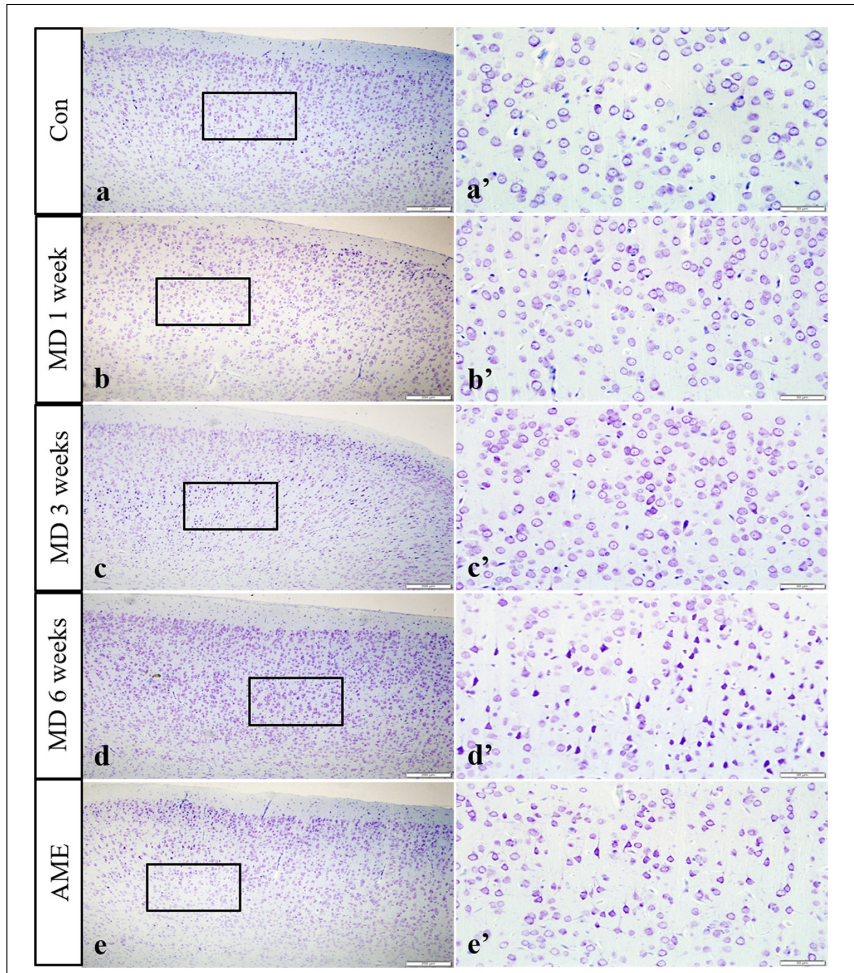

FIGURE 3 | Thionine staining of the cortex. Panels (a'-e') are magnified areas of panels (a-e), respectively. With increasing periods of morphine dependence, Nissl body structures are not clear, and neurons are pyknotic and deeply stained. Bars $=200 \mu \mathrm{m}$ in panels $(\mathbf{a}-\mathbf{e})$. Bars $=50 \mu \mathrm{m}$ in panels (a'-e'); MD, morphine dependence; AME, acute morphine exposure.

were stained brown, while NURR1 protein was localized to the nucleus and was stained red.

ANOVA for GRP78-positive cells in the cerebral cortex showed that there were significant differences among the groups $(F[4,25]=687.7 ; P<0.0001)$. Compared with the control group (5.24 $\pm 1.55, n=6)$, GRP78 expression was significantly upregulated in the 1-week MD $(38.63 \pm 3.51, P<0.01, n=6)$, 3-week MD (90.74 $\pm 6.25, P<0.01, n=6)$, 6-week MD $(77.52 \pm 5.15, P<0.01, n=6)$, and AME $(24.4 \pm 2.46, P<0.01$, $n=6$ ) groups (Figure 4).

ANOVA for p-eIF2 $\alpha$-positive cells in the cerebral cortex showed that there were significant differences among the groups $(F[4,25]=99.58 ; P<0.0001)$. Compared with the control group $(1.12 \pm 0.94, n=6)$, p-eIF2 $\alpha$ expression remained at a low level in the 6-week MD group $(1.45 \pm 1.34, n=6)$, but was significantly upregulated in the 1-week $\mathrm{MD}(10.42 \pm 2.41, P<0.01, n=6), 3$ week MD $(30.14 \pm 3.03, P<0.01, n=6)$, and AME $(11.62 \pm 3.20$, $P<0.01, n=6$ ) groups (Figure 5).

ANOVA for CHOP-positive cells in the cerebral cortex showed that there were significant differences among the groups ( $F[4$, $25]=1493 ; P<0.0001)$. Compared with the control group $(15.23 \pm 1.55, n=6)$, CHOP expression was significantly upregulated in the 1-week MD $(54.72 \pm 1.89, P<0.01, n=6)$, 3-week MD (114.12 $\pm 3.67, P<0.01, n=6)$, 6-week MD 

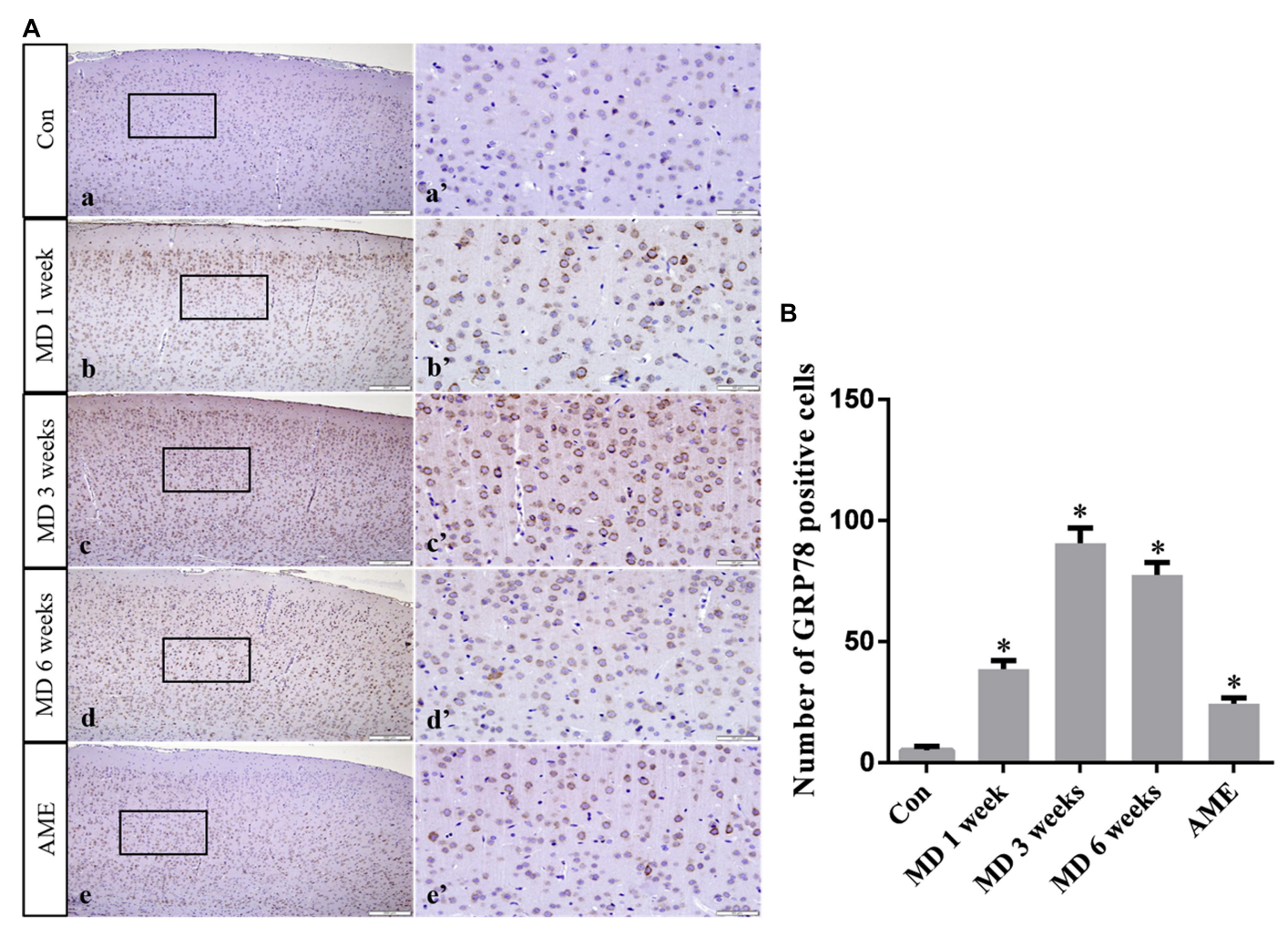

FIGURE 4 | (A) Representative images showing GRP78 immunohistochemistry in the cortex. Panels (a'-e') are magnified areas of panels (a-e). Bars = $200 \mu \mathrm{m}$ in panels (a-e). Bars $=50 \mu \mathrm{m}$ in panels (a'-e'). (B) Quantitative analysis of the number of GRP78 positive cells. The data are shown as mean \pm SD, ${ }^{\star} P<0.05$ vs. control group $(n=6)$. MD, morphine dependence; AME, acute morphine exposure.

$(56.46 \pm 1.43, P<0.01, n=6)$, and $\operatorname{AME}(34.46 \pm 1.55, P<0.01$, $n=6)$ groups (Figure 6).

ANOVA for ATF6-positive cells in the cerebral cortex showed that there were significant differences among the groups ( $F$ $[4,25]=257.5 ; P<0.0001)$. Compared with the control group (1.11 $\pm 1.14, n=6)$, ATF6 expression was significantly upregulated in the 1-week MD $(15.2 \pm 2.22, P<0.01, n=6)$, 3week MD (65.2 $\pm 5.07, P<0.01, n=6)$, 6-week MD (29.5 \pm 6.74 , $P<0.01, n=6)$, and AME $(38.34 \pm 4.57, P<0.01, n=6)$ groups (Figure 7).

ANOVA for NURR1-positive cells in the cerebral cortex showed that there were significant differences among the groups $(F[4,25]=8.613 ; P=0.0002)$. Compared with the control group $(9.12 \pm 0.88, n=6)$, NURR1 expression remained at a high level in the AME group $(8.71 \pm 1.25, n=6)$, but was significantly downregulated in the 1 -week $\mathrm{MD}(7.63 \pm 0.84, P<0.01, n=6)$, 3-week MD (7.21 $\pm 0.92, P<0.01, n=6)$, and 6-week MD $(6.94 \pm 0.74, P<0.01, n=6)$ groups (Figure 8).

\section{Ki-67 Expression Around the Ventricle}

Double-labeling showed that Ki-67 mostly co-localized with the neuronal marker MAP2 around the ventricle. Ki-67 protein was located in the nucleus and was stained red, and MAP2 protein was located in the cytoplasm and was stained green. ANOVA for Ki-67-positive cells in the cerebral cortex showed that there were significant differences among the groups $(F[4,25]=163.4$; $P<0.0001)$. Compared with the control group (44.32 \pm 3.81 , $n=6)$, the number of Ki-67/MAP2-positive cells remained at a high level in the AME group $(48.12 \pm 5.04, n=6)$, and was significantly downregulated in the 1 -week MD (35.62 \pm 2.95 , $P<0.01, n=6)$, 3-week MD (32.63 $\pm 3.17, P<0.01, n=6)$, and 6-week MD $(9.32 \pm 1.63, P<0.01, n=6)$ groups (Figure 9).

\section{DISCUSSION}

Several studies have shown that long-term MD can cause acute or chronic cerebral ischemia and hypoxia by directly affecting the central nervous system and inhibiting the respiratory center, which can lead to central nervous system damage (Brailoiu et al., 2004; Volkow and Skolnick, 2012). Animal models allow for the identification and investigation of mechanisms underlying the effects of MD. In the present study, we established rat models of AME and different durations of MD aiming to better understand the mechanisms involved in morphine exposure-induced injury on the body and provide treatment and prevention strategies.

The cerebral cortex comprises a layer of neurons and synapses located on the surface of the cerebral hemispheres. The cerebral cortex is folded into gyri, and approximately two-thirds of it is submerged inside brain fissures. The cortex is involved in higher mental functions, general movements, functions of the 

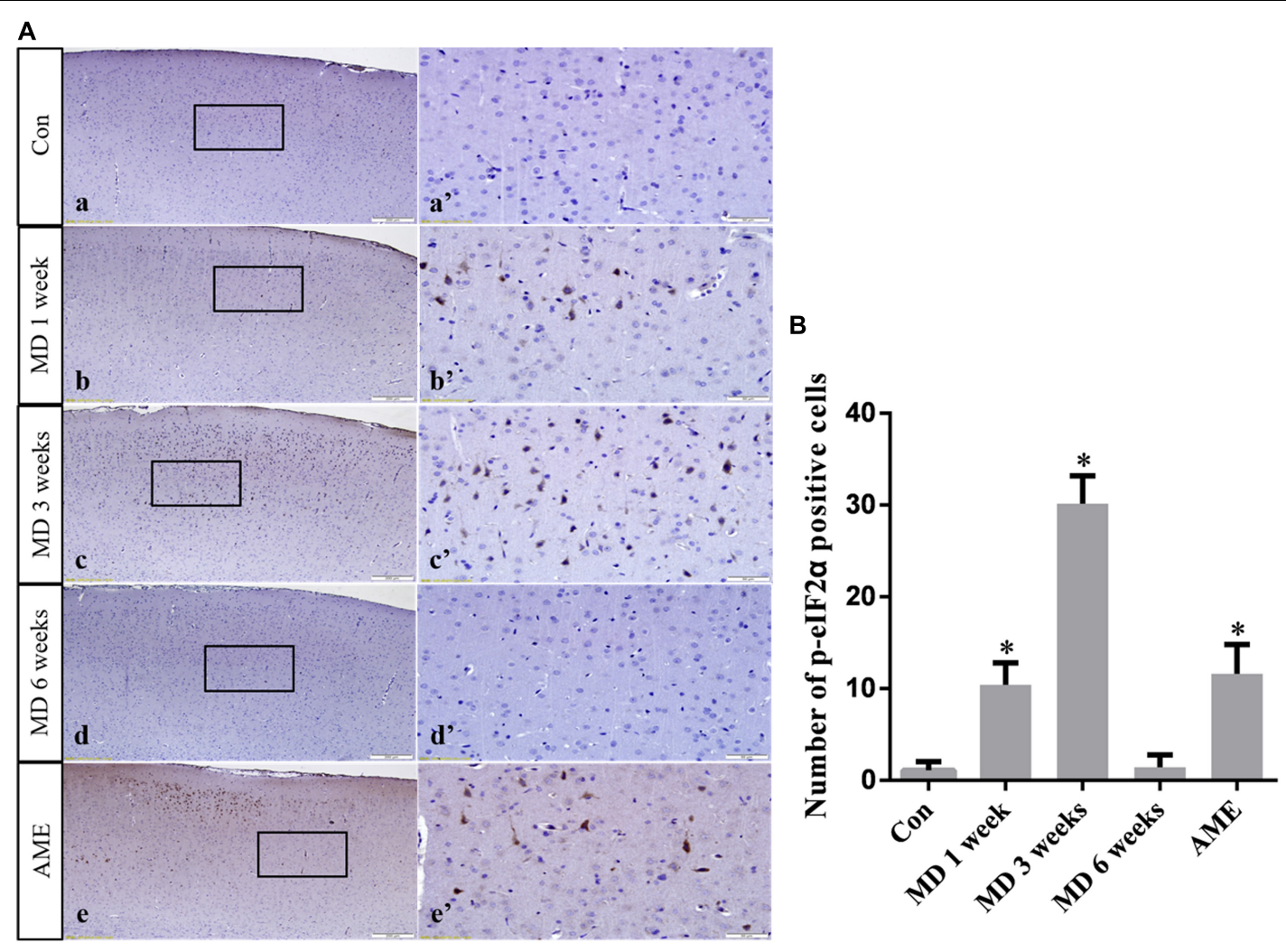

FIGURE 5 | (A) Representative images showing p-elF2 $\alpha$ immunohistochemistry in the cortex. Panels (a'-e') are magnified areas of panels (a-e). Bars = 200 $\mu$ m in panels (a-e). Bars $=50 \mu \mathrm{m}$ in panels (a'-e'). (B) Quantitative analysis of the number of p-elF2 $\alpha$ positive cells. The data are shown as mean $\pm \mathrm{SD},{ }^{*} P<0.05$ vs. control group $(n=6)$. MD, morphine dependence; AME, acute morphine exposure.

viscera, perception, and behavioral reactions (Cadwell et al., 2019). However, the changes induced in cortical neurons by MD or AME remain poorly understood. In the present study, the results of hematoxylin and eosin ( $\mathrm{H} \& \mathrm{E})$ and thionine staining revealed that long-term $\mathrm{MD}$ and $\mathrm{AME}$ resulted in pyknotic neurons and a reduction in the number of Nissl bodies, which suggested that long-term MD and AME can cause nerve damage, and that, with increasing periods of morphine exposure, nerve damage can become more extensive.

There is an important link between ERS and body damage. Several studies have demonstrated that ERS not only participates in the occurrence and progression of a variety of neurodegenerative diseases, but also plays a critical role in the pathology of nerve cell dysfunction (Johnson et al., 2011; Xiang et al., 2017; Muneer and Shamsher Khan, 2019). Under ERS, the protective mechanisms of the cell are activated, namely, the unfolded protein response (UPR), which results in the upregulation of the expression of GRP78, a molecular chaperone. GRP78 can bind to misfolded or unfolded proteins, thereby reducing the burden of the ER and restoring its function (Ni et al., 2011). In the present study, the expression level of GRP78 in the AME group was significantly higher than that in the control group, and, with increasing periods of morphine exposure, the expression level of GRP78 showed a significantly increasing trend. This suggested that ERS occurred in the cortex of morphine-dependent rats, and that the body's protective mechanisms were activated.

When morphine exposure persists, signaling pathways downstream of ERS will be activated, which can result in cell damage, or even cell death (Omura et al., 2013; Zhang et al., 2017). This may explain the shrinkage, disappearance of Nissl bodies, and other increasingly serious pathological impairments observed in this study.

Activating transcription factor 6 is a type-II transmembrane glycoprotein that, under ERS, can be transferred from the ER to the Golgi apparatus, where it is cleaved by site- 1 and site2 proteases. The $\mathrm{N}$-terminal fragment, containing the alkaline leucine zipper domain, translocates into the nucleus and acts as a transcription factor that can activate the expression of ERSrelated genes by combining ERS response elements and UPR elements (Papaioannou et al., 2018). Because the ERS response triggered by the selective activation of ATF6 is conducive to virus replication and the maintenance of cell viability, it is currently believed that the primary role of the ATF6 signaling pathway is the promotion of cell survival (Hillary and FitzGerald, 2018). In the present study, the expression level of ATF6 in the AME group was significantly higher than that in the control group, and, with increasing periods of morphine exposure, the expression level of ATF6 increased in the initial stages of MD, and then decreased at 6 weeks. These results showed that the protective 

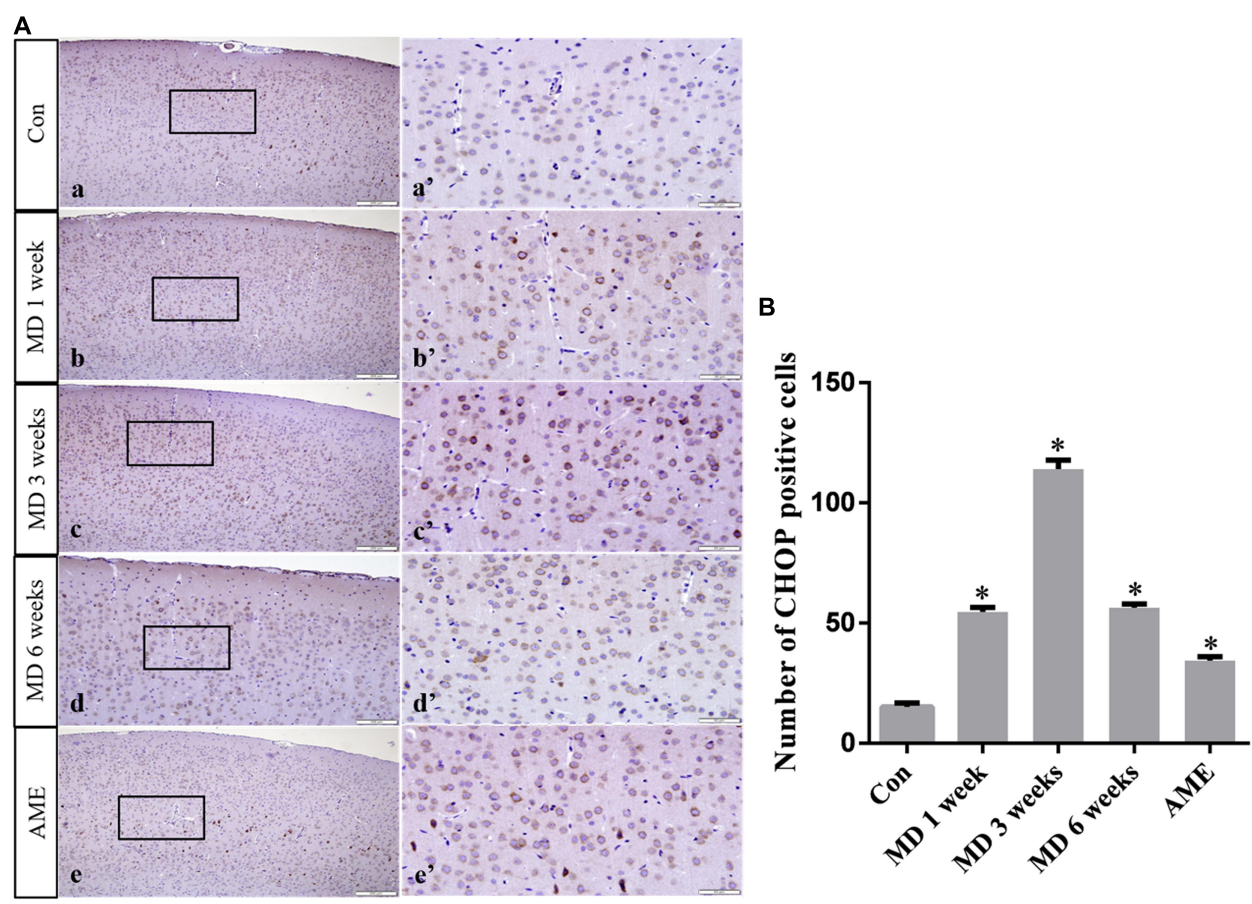

FIGURE 6 | (A) Representative images showing CHOP immunohistochemistry in the cortex. Panels (a'-e') are magnified areas of panels (a-e). Bars = $200 \mu \mathrm{m}$ in panels (a-e). Bars $=50 \mu \mathrm{m}$ panels in (a'-e'). (B) Quantitative analysis of the number of CHOP positive cells. The data are shown as mean \pm SD, * $P<0.05$ vs. control group $(n=6)$. MD, morphine dependence; AME, acute morphine exposure.

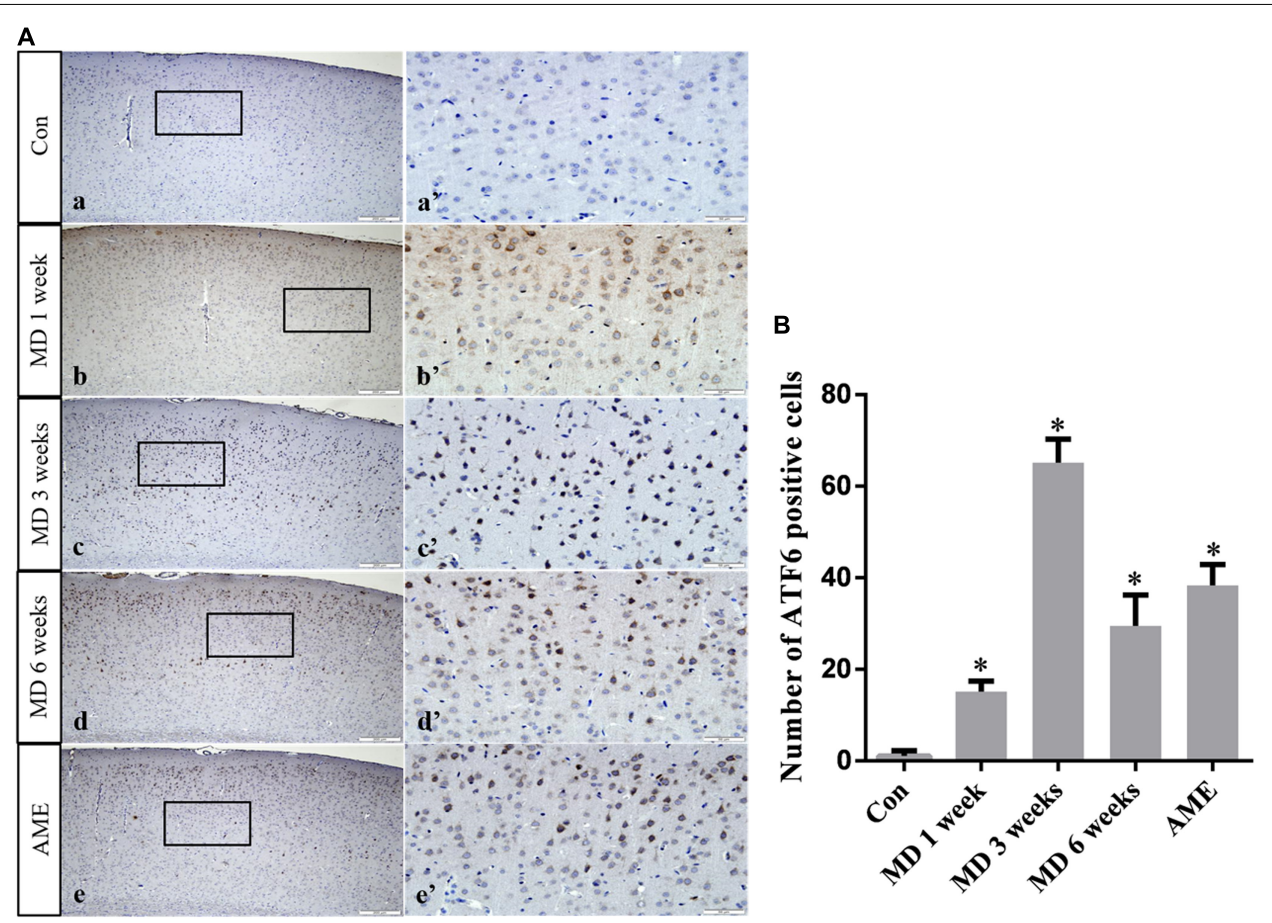

FIGURE 7 | (A) Representative images showing ATF6 immunohistochemistry in the cortex. Panels (a'-e') are magnified areas of panels (a-e). Bars = 200 $\mu$ m in panels (a-e). Bars $=50 \mu \mathrm{m}$ in panels (a'-e'). (B) Quantitative analysis of the number of ATF6 positive cells. The data are shown as mean \pm SD, ${ }^{*} P<0.05$ vs. control group $(n=6)$. MD, morphine dependence; AME, acute morphine exposure. 

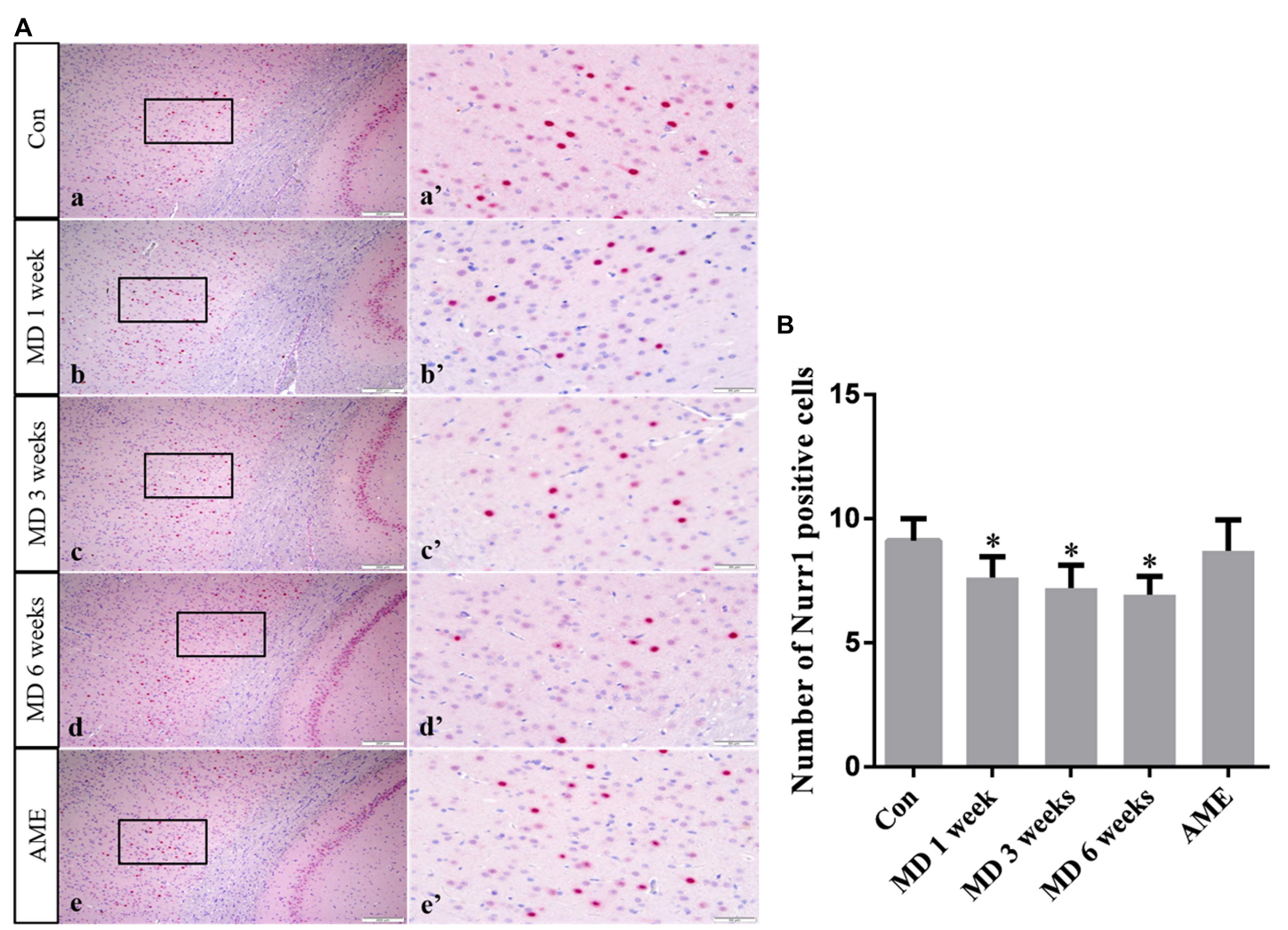

FIGURE 8 | (A) Representative images showing Nurr1 immunohistochemistry in the cortex. Panels (a'-e') are magnified areas of panels (a-e). Bars = $200 \mu \mathrm{m}$ in panels (a-e). Bars $=50 \mu \mathrm{m}$ in panels (a'-e'). (B) Quantitative analysis of the number of Nurr1 positive cells. The data are shown as mean \pm SD, $* P<0.05$ vs. control group $(n=6)$. MD, morphine dependence; AME, acute morphine exposure.

effect of ATF6 on cortical neurons was significantly weakened with prolonged morphine exposure, leading to the activation of a series of downstream signaling pathways that induced cell injury and/or cell death.

CHOP, also known as growth arrest and DNA-damage inducible gene 153 (GADD153), is a key, ERS-specific proapoptotic transcription factor. Under normal physiological conditions, CHOP is expressed at a very low level (Yi et al., 2019). The PERK/eIF2 $\alpha$ signaling pathway plays a dominant role in inducing CHOP expression (Rozpedek et al., 2016). PERK can inhibit the effect of eIF2B by promoting the phosphorylation of eIF $2 \alpha$, which can reduce protein translation and the amount of unfolded protein, thereby maintaining cell survival. When ERS is severe or prolonged, upstream open reading frame regulatory sequences present in the 5'-untranslated regions of mRNAs become activated, which inhibits eIF $2 \alpha$-dependent protein translation and activates the downstream ATF4/CHOP signaling pathway. Continuous expression of CHOP can cause cell damage, or even cell death (Cubillos-Ruiz et al., 2017; Wu et al., 2018; Lorenzon-Ojea et al., 2020). In this study, the expression levels of p-eIF2 $\alpha$ and CHOP in the AME group were significantly higher than those of the control group. With increasing periods of morphine exposure, the expression level of p-eIF $2 \alpha$ first increased, and then underwent a significant decline at 6 weeks, while CHOP showed sustained high levels of expression. These results suggested that the protective and damaging effects of ERS on neurons were in a state of mutual restraint in the 1- and 3-week MD groups. Subsequently, the high level of CHOP expression could lead to cell injury and death, which was consistent with our observations of pathological changes in cortical neurons after 6 weeks of MD. These data indicated that the PERK/p-eIF $2 \alpha / \mathrm{CHOP}$ pathway is associated with morphine exposure-induced cortical neuronal injury.

$\mathrm{Ki}-67$ is a nuclear antigen specifically expressed by proliferating cells and is commonly used to evaluate tumor proliferative ability, malignancy, and prognosis (Scholzen and Gerdes, 2000; Miller et al., 2018). Under normal circumstances, $\mathrm{Ki}-67$ is expressed in multiple brain regions, especially around the ventricle. Given that Ki-67-positive cells around the ventricle are NSCs and neural progenitor cells (NPCs), the expression of Ki-67 likely reflects the proliferation of nerve cells. In the present study, double immunofluorescence labeling of the neuron-specific marker MAP2 and Ki-67 were used as indicators of the proliferative status of NSCs and NPCs around the lateral ventricle after morphine exposure. No significant differences were observed between the AME and control groups, while the number of double-labeled Ki-67/MAP2-positive cells around the lateral ventricle showed a decreasing trend with increasing periods of morphine exposure. These results suggested that MD suppressed the proliferation of NSCs and NPCs around the 


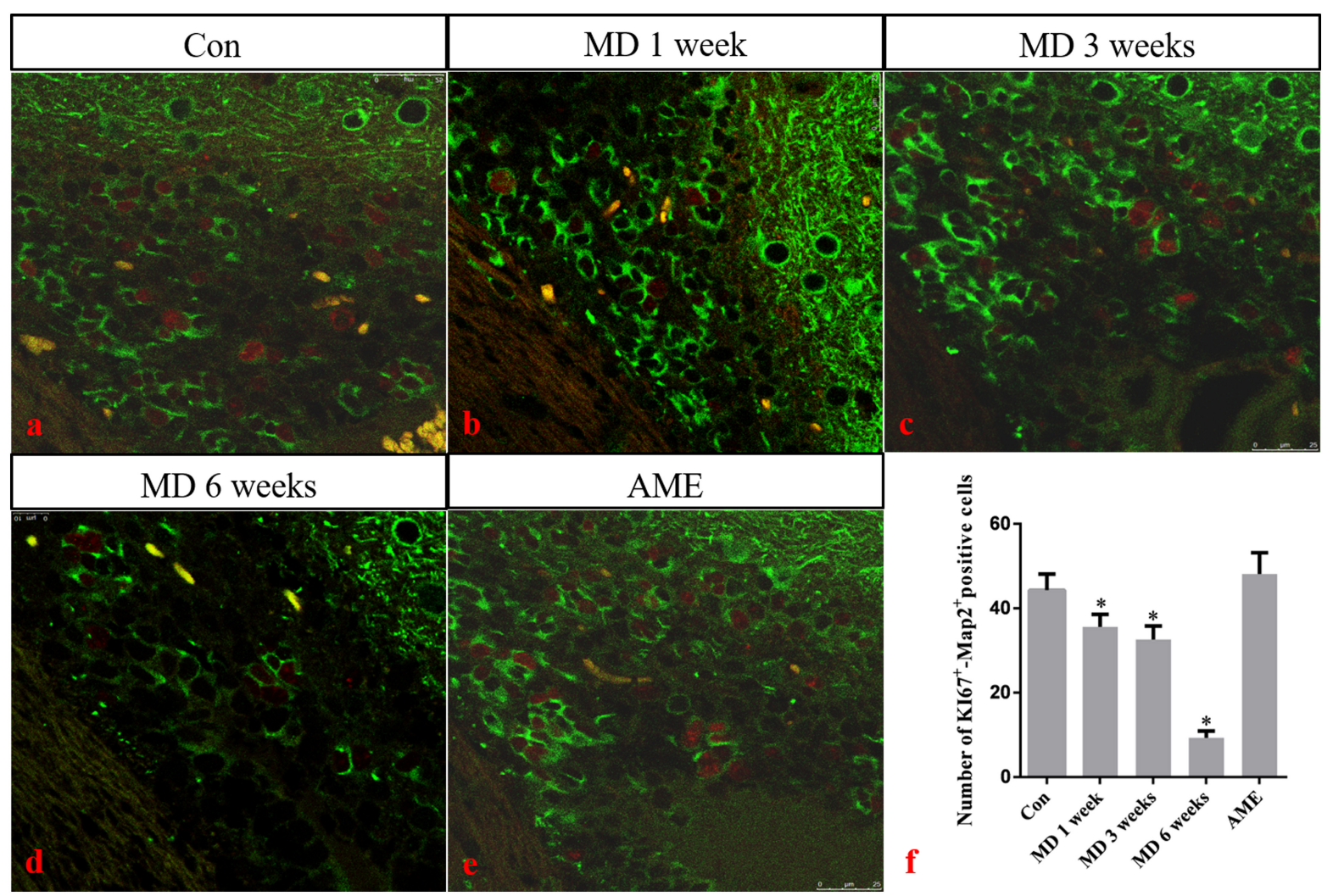

FIGURE 9 | (a-e) Immunofluorescence staining for Ki67 (red) and Map2 (green) in the cortex. Bars = $25 \mu \mathrm{m}$ in panels (a-e). (f) Quantitative analysis of the number of Ki67+ - Map2+ positive cells. The data are shown as mean \pm SD, ${ }^{*} P<0.05$ vs. control group $(n=6)$. MD, morphine dependence; AME, acute morphine exposure.

lateral ventricle. We have previously shown that NURR1 plays an important role in the differentiation, migration, and maturation of atypical dopaminergic neurons outside the midbrain ( $\mathrm{Li}$ et al., 2011, 2012). Although NURR1 is mainly expressed in dopaminergic neurons in the ventral side of the midbrain, it is also expressed in layers II-V of the cortex (Sano et al., 2008). In the present study, NURRl expression was significantly decreased in the morphine-dependent groups. These results suggested that MD inhibited the differentiation, migration, and maturation of cortical neurons, which may result in a decrease in the number of newborn neurons.

In conclusion, the results of this study demonstrated that ERS is involved in morphine exposure-induced injury. Moreover, morphine exposure inhibited the proliferation of endogenous NSCs and the differentiation and maturation of newborn neurons, thereby affecting nerve repair. We believe these findings provide morphological evidence for the mechanisms involved in morphine-induced cortical neuron injury and the repair process after brain injury.

\section{DATA AVAILABILITY STATEMENT}

The original contributions presented in the study are included in the article/supplementary material, further inquiries can be directed to the corresponding author/s.

\section{ETHICS STATEMENT}

The animal study was reviewed and approved by the Institutional Review Board for Animal Experiments at the Hebei Medical University.

\section{AUTHOR CONTRIBUTIONS}

JL and SY designed and performed the experiments. WS and GZ performed the statistical analysis and organized the data. SW and QQ created the figures. BC and YL supervised the research design and revised the manuscript. All the authors read and approved the final version of the manuscript.

\section{FUNDING}

This study was supported by the National Natural Science Foundation of China (81971787), the Key Projects of Basic Research in Hebei Province (189677108D), the Natural Science Foundation of Hebei Province (H2020206150), the Science and Technology Research Project of Hebei Higher Education Institutions (QN2019095), and the Spring Rain Project of Hebei Medical University (CYQD201905). 


\section{REFERENCES}

Adedayo, A. D., Aderinola, A. A., Adekilekun, T. A., Olaolu, O. O., Olanike, A. M., and Olayemi, I. K. (2018). Morphine-alcohol treatment impairs cognitive functions and increases neuro-inflammatory responses in the medial prefrontal cortex of juvenile male rats. Anat. Cell Biol. 51, 41-51. doi: 10.5115/acb.2018. 51.1 .41

Brailoiu, E., Hoard, J., Brailoiu, G. C., Chi, M., Godbolde, R., and Dun, N. J. (2004). Ultra low concentrations of morphine increase neurite outgrowth in cultured rat spinal cord and cerebral cortical neurons. Neurosci. Lett. 365, 10-13. doi: 10.1016/j.neulet.2004.03.092

Cadwell, C. R., Bhaduri, A., Mostajo-Radji, M. A., Keefe, M. G., and Nowakowski, T. J. (2019). Development and arealization of the cerebral cortex. Neuron 103, 980-1004. doi: 10.1016/j.neuron.2019.07.009

Cubillos-Ruiz, J. R., Mohamed, E., and Rodriguez, P. C. (2017). Unfolding antitumor immunity: ER stress responses sculpt tolerogenic myeloid cells in cancer. J Immunother. Cancer 5:5. doi: 10.1186/s40425-016-0203-4

Dai, Y., Sun, F., Zhu, H., Liu, Q., Xu, X., Gong, P., et al. (2019). Effects and mechanism of action of neonatal versus adult astrocytes on neural stem cell proliferation after traumatic brain injury. Stem Cells 37, 1344-1356. doi: 10. 1002/stem.3060

Dong, J., Li, S., Mo, J. L., Cai, H. B., and Le, W. D. (2016). Nurr1-based therapies for Parkinson's disease. CNS Neurosci. Ther. 22, 351-359. doi: 10.1111/cns. 12536

Hillary, R. F., and FitzGerald, U. (2018). A lifetime of stress: ATF6 in development and homeostasis. J. Biomed. Sci. 25:48. doi: 10.1186/s12929-018-0453-1

$\mathrm{Hu}, \mathrm{H}$. , Tian, M., Ding, C., and Yu, S. (2019). The C/EBP homologous protein (CHOP) transcription factor functions in endoplasmic reticulum stressinduced apoptosis and microbial infection. Front. Immunol. 9:3083. doi: 10. 3389/fimmu.2018.03083

Ibrahim, I. M., Abdelmalek, D. H., and Elfiky, A. A. (2019). GRP78: a cell's response to stress. Life Sci. 226, 156-163. doi: 10.1016/j.lfs.2019.04.022

Iurlaro, R., and Muñoz-Pinedo, C. (2016). Cell death induced by endoplasmic reticulum stress. FEBS J. 283, 2640-2652. doi: 10.1111/febs.13598

Johnson, G. G., White, M. C., and Grimaldi, M. (2011). Stressed to death: targeting endoplasmic reticulum stress response induced apoptosis in gliomas. Curr. Pharmac. Design 17, 284-292. doi: 10.2174/138161211795049660

Katebi, S. N., Razavi, Y., Zeighamy Alamdary, S., Khodagholi, F., and Haghparast, A. (2013). Morphine could increase apoptotic factors in the nucleus accumbens and prefrontal cortex of rat brain's reward circuitry. Brain Res. 1540, 1-8. doi: 10.1016/j.brainres.2013.09.045

Kropski, J. A., and Blackwell, T. S. (2018). Endoplasmic reticulum stress in the pathogenesis of fibrotic disease. J. Clin. Invest. 128, 64-73. doi: 10.1172/ JCI93560

Lamb, R. J., and Ginsburg, B. C. (2018). Addiction as a BAD, a behavioral allocation disorder. Pharmacol. Biochem. Behav. 164, 62-70. doi: 10.1016/j.pbb.2017. 05.002

Li, Y., Cong, B., Ma, C., Qi, Q., Fu, L., Zhang, G., et al. (2011). Expression of Nurr1 during rat brain and spinal cord development. Neurosci. Lett. 488, 49-54. doi: 10.1016/j.neulet.2010.10.078

Li, Y., Qi, Q., Cong, B., Shi, W., Liu, X., Zhang, G., et al. (2012). Expression patterns of Nurr1 in rat retina development. J. Mol. Histol 43, 633-639. doi: 10.1007/s10735-012-9433-z

Lorenzon-Ojea, A. R., Yung, H. W., Burton, G. J., and Bevilacqua, E. (2020). The potential contribution of stromal cell-derived factor 2 (SDF2) in endoplasmic reticulum stress response in severe preeclampsia and labor-onset. Biochim. Biophys. Acta Mol. Basis Dis. 1866:165386. doi: 10.1016/j.bbadis.2019.01.012

Luo, F. C., Zhao, L., Deng, J., Liang, M., Zeng, X. S., Liu, H., et al. (2013). Geranylgeranylacetone protects against morphine-induced hepatic and renal damage in mice. Mol. Med. Rep. 7, 694-700. doi: 10.3892/mmr.2012.1217

Maldonado, R., Negus, S., and Koob, G. F. (1992). Precipitation of morphine withdrawal syndrome in rats by administration of mu-, delta- and kappaselective opioid antagonists. Neuropharmacology 31, 1231-1241. doi: 10.1016/ 0028-3908(92)90051-p

Miller, I., Min, M., Yang, C., Tian, C., Gookin, S., Carter, D., et al. (2018). Ki67 is a Graded rather than a binary marker of proliferation versus quiescence. Cell Rep. 24, 1105-1112.e5. doi: 10.1016/j.celrep.2018.06.110
Muneer, A., and Shamsher Khan, R. M. (2019). Endoplasmic Reticulum stress: implications for neuropsychiatric disorders. Chonnam. Med. J. 55, 8-19. doi: 10.4068/cmj.2019.55.1.8

Ni, M., Zhang, Y., and Lee, A. S. (2011). Beyond the endoplasmic reticulum: atypical GRP78 in cell viability, signalling and therapeutic targeting. Biochem. J. 434, 181-188. doi: 10.1042/BJ20101569

Omura, T., Asari, M., Yamamoto, J., Oka, K., Hoshina, C., Maseda, C., et al. (2013). Sodium tauroursodeoxycholate prevents paraquat-induced cell death by suppressing endoplasmic reticulum stress responses in human lung epithelial A549 cells. Biochem. Biophys. Res. Commun. 432, 689-694. doi: 10.1016/j.bbrc. 2013.01.131

Papaioannou, A., Higa, A., Jégou, G., Jouan, F., Pineau, R., Saas, L., et al. (2018). Alterations of EDEM1 functions enhance ATF6 pro-survival signaling. FEBS J. 285, 4146-4164. doi: 10.1111/febs.14669

Paxinos, G., and Watson, C. (2007). The Rat Brain in Stereotaxic Coordinates, 6 Edn. Amsterdam: Academic Press.

Rohbani, K., Sabzevari, S., Sadat-Shirazi, M. S., Nouri Zadeh-Tehrani, S., Ashabi, G., Khalifeh, S., et al. (2019). Parental morphine exposure affects repetitive grooming actions and marble burying behavior in the offspring: Potential relevance for obsessive-compulsive like behavior. Eur. J. Pharmacol. 865:172757. doi: 10.1016/j.ejphar.2019.172757

Rozpedek, W., Pytel, D., Mucha, B., Leszczynska, H., Diehl, J. A., and Majsterek, I. (2016). The role of the PERK/eIF2 $\alpha /$ ATF4/CHOP Signaling pathway in tumor progression during endoplasmic reticulum stress. Curr. Mol. Med. 16, 533-544. doi: 10.2174/1566524016666160523143937

Sano, K., Miyaji-Yamaguchi, M., Tsutsui, K. M., and Tsutsui, K. (2008). Topoisomerase IIbeta activates a subset of neuronal genes that are repressed in AT-rich genomic environment. PLoS One 3:e4103. doi: 10.1371/journal.pone. 0004103

Scholzen, T., and Gerdes, J. (2000). The Ki-67 protein: from the known and the unknown. J. Cell Physiol. 182, 311-322. doi: 10.1002/(SICI)1097-4652(200003) 182:3<311::AID-JCP1<3.0.CO;2-9

Shi, W., Zhang, Y., Zhao, G., Wang, S., Zhang, G., Ma, C., et al. (2019). Dysregulation of dopaminergic regulatory factors TH, Nurr1, and Pitx3 in the ventral tegmental area associated with neuronal injury induced by chronic morphine dependence. Int. J. Mol. Sci. 20:250. doi: 10.3390/ijms2002 0250

Volkow, N. D., and Skolnick, P. (2012). New medications for substance use disorders: challenges and opportunities. Neuropsychopharmacology 37, 290292. doi: 10.1038/npp.2011.84

Wu, F., Qiu, J., Fan, Y., Zhang, Q., Cheng, B., Wu, Y., et al. (2018). Apelin13 attenuates ER stress-mediated neuronal apoptosis by activating G $\alpha \mathrm{i} / \mathrm{G} \alpha \mathrm{q}$ CK2 signaling in ischemic stroke. Exp. Neurol. 302, 136-144. doi: 10.1016/j. expneurol.2018.01.006

Xiang, C., Wang, Y., Zhang, H., and Han, F. (2017). The role of endoplasmic reticulum stress in neurodegenerative disease. Apoptosis 22, 1-26. doi: 10.1007/ s10495-016-1296-4

Yi, S., Chen, K., Zhang, L., Shi, W., Zhang, Y., Niu, S., et al. (2019). Endoplasmic reticulum stress is involved in stress-induced hypothalamic neuronal injury in rats via the PERK-ATF4-CHOP and IRE1-ASK1-JNK pathways. Front. Cell Neurosci. 13:190. doi: 10.3389/fncel.2019.00190

Zhang, M., Liu, X., Wang, Q., Ru, Y., Xiong, X., Wu, K., et al. (2017). NDRG2 acts as a PERK co-factor to facilitate PERK branch and ERSinduced cell death. FEBS Lett. 591, 3670-3681. doi: 10.1002/1873-3468. 12861

Conflict of Interest: The authors declare that the research was conducted in the absence of any commercial or financial relationships that could be construed as a potential conflict of interest.

Copyright (c) $2021 \mathrm{Liu}, \mathrm{Yi}$, Shi, Zhang, Wang, Qi, Cong and Li. This is an open-access article distributed under the terms of the Creative Commons Attribution License (CC BY). The use, distribution or reproduction in other forums is permitted, provided the original author(s) and the copyright owner(s) are credited and that the original publication in this journal is cited, in accordance with accepted academic practice. No use, distribution or reproduction is permitted which does not comply with these terms. 